\title{
Successful salvage therapy with Daptomycin for osteomyelitis caused by methicillin-resistant Staphylococcus aureus in a renal transplant recipient with Fabry-Anderson disease
}

\author{
Ennio Polilli', Tamara Ursini ${ }^{2}$, Elena Mazzotta ${ }^{3}$, Federica Sozio ${ }^{3}$, Vincenzo Savini ${ }^{1}$, Domenico D'Antonio ${ }^{1}$, \\ Michelino Barbato ${ }^{4}$, Augusta Consorte ${ }^{3}$ and Giustino Parruti ${ }^{3,5^{*}}$
}

\begin{abstract}
Daptomycin is licensed in adults for the management of Staphylococcus aureus methicillin-resistant infections, including bone and skin complicated infections. We describe for the first time its use in a renal transplant recipient for Fabry-Anderson Disease with right heel osteomyelitis. The patient was unresponsive to first-line Teicoplanin and second-line Tigecycline, whereas he was successfully treated with third-line Daptomycin monotherapy at $4 \mathrm{mg} / \mathrm{Kg} /$ qd for 4 weeks. Local debridement was performed in advance of each line of treatment.
\end{abstract}

Keywords: Methicillin-resistant Staphylococcus aureus, Osteomyelitis, Daptomycin, Salvage therapy, Antibiotic therapy

\section{Background}

Vancomycin, the first-line antibiotic choice for bone and joint infections caused by grampositive bacteria, has been reported with declining rates of efficacy against methicillin resistant Staphylococcus aureus (MRSA) isolates [1]. The treatment of osteomyelitis, septic arthritis and prosthetic joint infections with Vancomycin can therefore be difficult, often requiring prolonged administration [2-5]. The reduced susceptibility of S. aureus to Vancomycin may be partially due to biofilms, facilitating bacterial persistence [6-8]. Daptomycin, presently considered as a reliable alternative to the class of glycopeptides in these conditions [9], was recently used successfully, alone or in combination, for osteomyelitis caused by gram-positive pathogens including MRSA, unresponsive to other antibiotics [10]. Dosing of Daptomycin, however, and its ability to penetrate into inflamed target tissues are still a matter of controversy [11]. In this report we describe for the first time a case of right hell osteomyelitis caused by MRSA and

\footnotetext{
* Correspondence: parruti@tin.it

${ }^{3}$ Infectious Disease Unit, Pescara General Hospital, Pescara, Italy

Full list of author information is available at the end of the article
}

successfully treated with third-line Daptomycin in a renal transplant recipient for Fabry-Anderson Disease (FAD).

\section{Case presentation}

A 38-year-old patient, diagnosed with FAD in 1990, was put on hemodialysis since 1999 due to end-stage renal insufficiency and received a kidney transplant in 2007. Renal function did not recover immediately after renal transplantation, and sequential renal biopsies documented early rejection. For this reason, the patient was put on high dose oral Cyclosporine (150 mg twice daily) and was treated with high dose steroids without benefit. Everolimus ( $2.5 \mathrm{mg} /$ daily) was therefore added and steroids tapered. Renal function improved 4 months after transplantation; hemodialysis was continued until that time. Due to relapsing decreases in renal function after each attempt to interrupt Everolimus, the association of these drugs could not be stopped ever since, although Cyclosporine could be reduced to $100 \mathrm{mg}$ twice daily after 1 year. Levels of both drugs were frequently monitored, falling in the therapeutic range throughout follow-up without further adjustments. Additional drugs prescribed after transplantation were: Cardioaspirin, 100 
mg/daily; Allopurinol $100 \mathrm{mg} /$ daily; Carvedilol $25 \mathrm{mg} /$ daily, Furosemide $25 \mathrm{mg} /$ daily, Replagal ${ }^{\circledR}$ (Agalsidase $\alpha$ ) 3 times/weekly until hospitalization. During hemodialysis, he suffered several traumatic and ischemic bone lesions; a Dual-energy X-ray absorptiometry (DEXA) scan, performed in 2006, revealed diffuse osteopenia, in spite of intact parathyroid glands and normal parallel parathormone levels.

In March, 2008, the patient was assisted in our ward due to a large pulmonary infiltrate and treated with i.v. Amoxicillin/Clavulanate and Fluconazole. The next month he was hospitalized in the orthopedic ward of Ortona Hospital (Chieti, Italy) because of an ulcerative lesion at his right heel with redness, swelling and the presence of necrotic tissue (Figure 1a). He was apyretic, with normal white and red cell counts and near-normal renal function tests, the estimated glomerular filtration rate ranging between 56 and $83 \mathrm{~mL} / \mathrm{min} / 1.73 \mathrm{~m}^{2}$ during hospitalization, as calculated using the CKD-EPI formula. Standard Rx scans of his right foot documented a detachment of a wide fragment of his cortical spongiosa in the lateral malleolus (Figure 1b). Cultural examination of wound essudates did not yield any isolate. The patient underwent extensive debridement of necrotic tissues and was put on hyperbaric oxygen therapy and Teicoplanin $200 \mathrm{mg}$ i.v. for 4 weeks, without benefit. The dose of Teicoplanin was decided by the assisting orthopedists at that time, the fear of renal overload causing
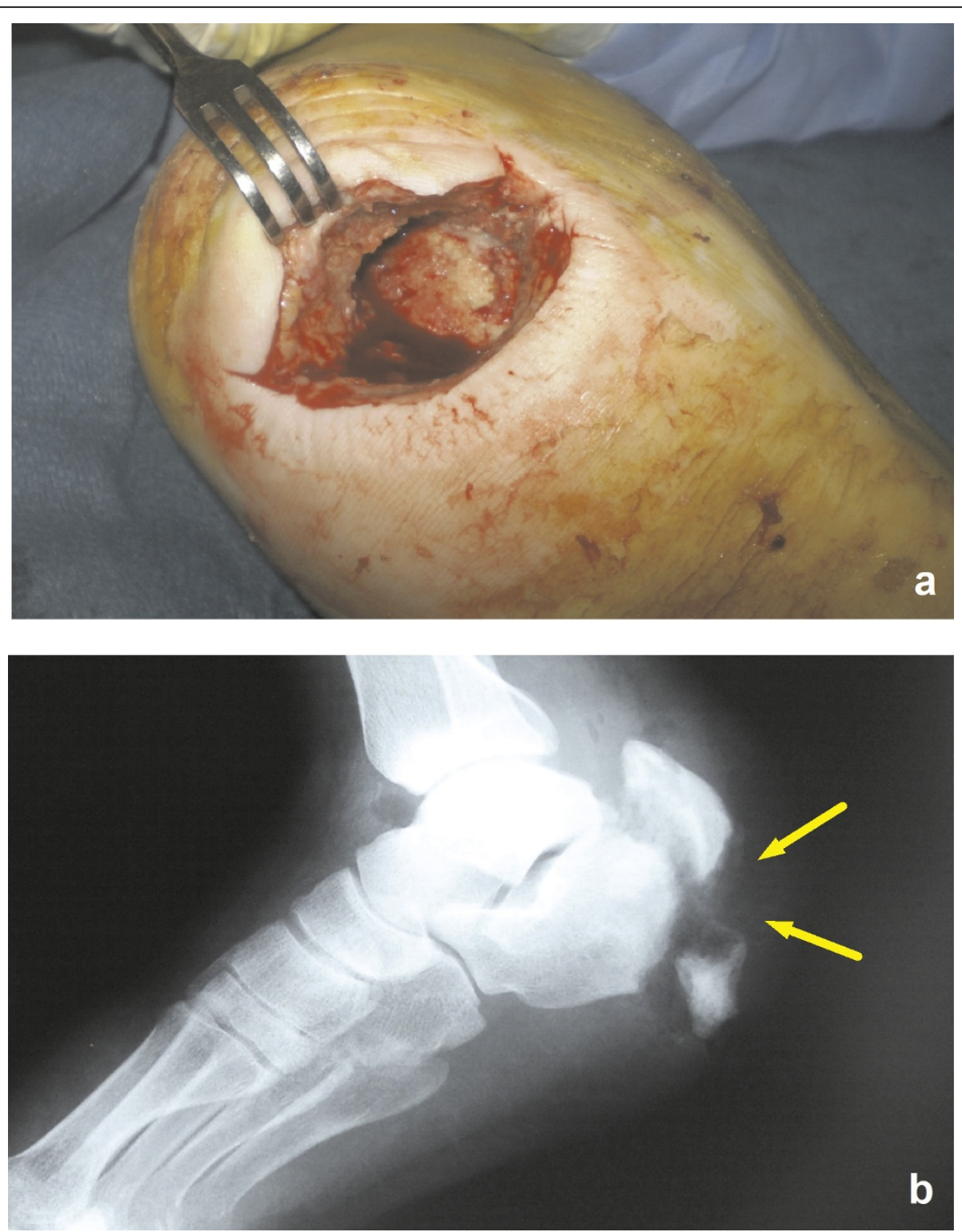

Figure 1 a) Ulcerative lesion of the right heel; b) Standard Rx scan of the right heel at patient entry. 
patent underdosing. In July, 2008, the patient was again evaluated in the Infectious Disease Unit of Pescara General Hospital for persistent fever and localized pain. Inflammation indexes were remarkably altered. A second debridement procedure was requested; cultures of debris grew several Streptococcus agalactiae isolates. To avoid renal overload, treatment with i.v. Tigecycline, 50 mg daily twice daily, together with Metronidazole, 500 mg three times daily, was prescribed. The patient was still assisted at the orthopedic ward of Ortona Hospital after consultation; a double daily access was arranged for him as outpatient, and the prescription of Metronidazole reduced to $500 \mathrm{mg}$ twice daily. A slight local improvement was noted, but relapsing fever ensued after 30 days of treatment. ESR rose to $112 \mathrm{~mm}$, CRP to $85 \mathrm{mg} / \mathrm{L}$. A focused CT scan of his right heel confirmed the presence of a fracture of his right calcaneus with fragmentation and irregular thickening of fracture edges, tarsal bone loss, gaseous components in neighboring soft tissues, abnormal spacing between calcaneus and cuboid. A few days later, the patient underwent a third debridement procedure. Culture specimens grew MRSA isolates, sensitive to Vancomycin $(\mathrm{MIC}=0.5 \mathrm{mg} / \mathrm{L})$ and Daptomycin. Based on these findings, i.v. Daptomycin (4 $\mathrm{mg} / \mathrm{kg}$ daily) was started, and administered for 4 weeks. CRP levels had dropped to $56 \mathrm{mg} / \mathrm{L}$ at this time. CPK levels during treatment were frequently monitored and found unmodified. At the end of treatment, deep palpation of the lesion did not cause pain; accompanying skin lesions had resolved; liver and renal function tests were normal, ESR was $50 \mathrm{~mm}$ and CRP 6,5 $\mathrm{mg} / \mathrm{L}$. Standard Rx scans and focused control tomography of the heel documented a reduction of bone resorption at affected segments, indicating effective control of osteomyelitis (Figure 2). The patient was discharged, and antibiotic therapy was continued at home with oral Trimetoprim/ Sulfamethoxazole (1 double strength tablet twice daily) and Doxycycline (100 mg twice daily) for 4 additional weeks, with frequent monitoring of hematological parameters, immunosuppressant drugs and renal function tests. No signs of toxicity were noted; the estimated value of GFR ranged between 63 and $85 \mathrm{~mL}$ using the CKD-EPI formula. At the end of treatment, the patient could walk with the support of crutches. In February, 2009, a complete normalization of inflammation indices was documented.

\section{Discussion}

Bone and joint discomfort is a common problem after transplantation. It is now well known that skeletal abnormalities, especially osteopenia with subsequent fractures, may develop following kidney transplantation $[12,13]$, osteomyelitis being one of the complications of fractures [14]. The complex impairment of bone metabolism due to FAD and long lasting hemodialysis in our patient may well have played a role in generating his recalcitrant infection [15]. At our best knowledge, here we report for the first time that Daptomycin in monotherapy at low dose $(4 \mathrm{mg} / \mathrm{Kg} /$ daily $)$ was safe and efficacious as a third line treatment for MRSA osteomyelitis of the heel in a renal transplant recipient on long-term immune suppression with Cyclosporine and Everolimus. Appropriate debridement of the lesion was performed in this patient before each of his treatment lines. No isolate was generated after the first procedure, and Staphylococcus osteomyelitis was postulated as a complication of his previous pneumonia. Vancomycin sensitivity of $S$. aureus was preserved until recently [16]; Vancomycin, however, has poor bone penetration and was unable to sterilize bone infections in animal models $[17,18]$. In spite of recent reports on its efficacy in human osteomyelitis at higher doses, the use of Vancomycin in our transplanted patient with ensuing full dose immunosuppressants was worrisome in the absence of a microbiological isolate, as the high recommended trough levels for Vancomycin (15-20 mg/L) in osteomyelitis might indeed have harmed the renal graft [19]. Low dose Teicoplanin was then preferred. Tigecycline plus Metronidazole were chosen after the second debridement because of the isolates of Streptococcus agalactiae obtained at that time. The etiologic role of these bacteria, however, may be questioned also in view of treatment failure. MRSA infection was finally documented by specimens cultured at the third debridement procedure. Monotherapy with Daptomycin for the following 4 weeks, at the cautious dose of $4 \mathrm{mg} / \mathrm{Kg} /$ daily, was preferred to Vancomycin. Long term administration of Linezolid, which tested equally effective and could have been given on an outpatient basis as oral therapy, was reckoned as potentially associated with worrisome side effects in this patient; furthermore, it is not permitted for treatment over 21 days [20]. Daptomycin is presently considered as an attractive alternative to glycopeptides in these cases, because of its ability to penetrate into inflamed subcutaneous adipose tissue and bones; in our patient it induced a rapid bone and wound recovery, with reversal of inflammation indexes [11]. The potency of Daptomycin has been demonstrated against a wide range of aerobic and anaerobic Gram-positive bacteria, including MRSA, glycopeptide-intermediate S. aureus, methicillin-resistant Coagulase-negative Staphylococci, and Vancomycinresistant Enterococcus (VRE) in bacteremias and endocarditis [21,22], skin and skin structure infection [23] and bone infections [10]. Daptomycin is safe with any state of renal function and dosing guidelines are available for hemodialysis patients [24] as well as for ICU patients with acute renal failure [25]. The characteristics and outcomes of patients with 


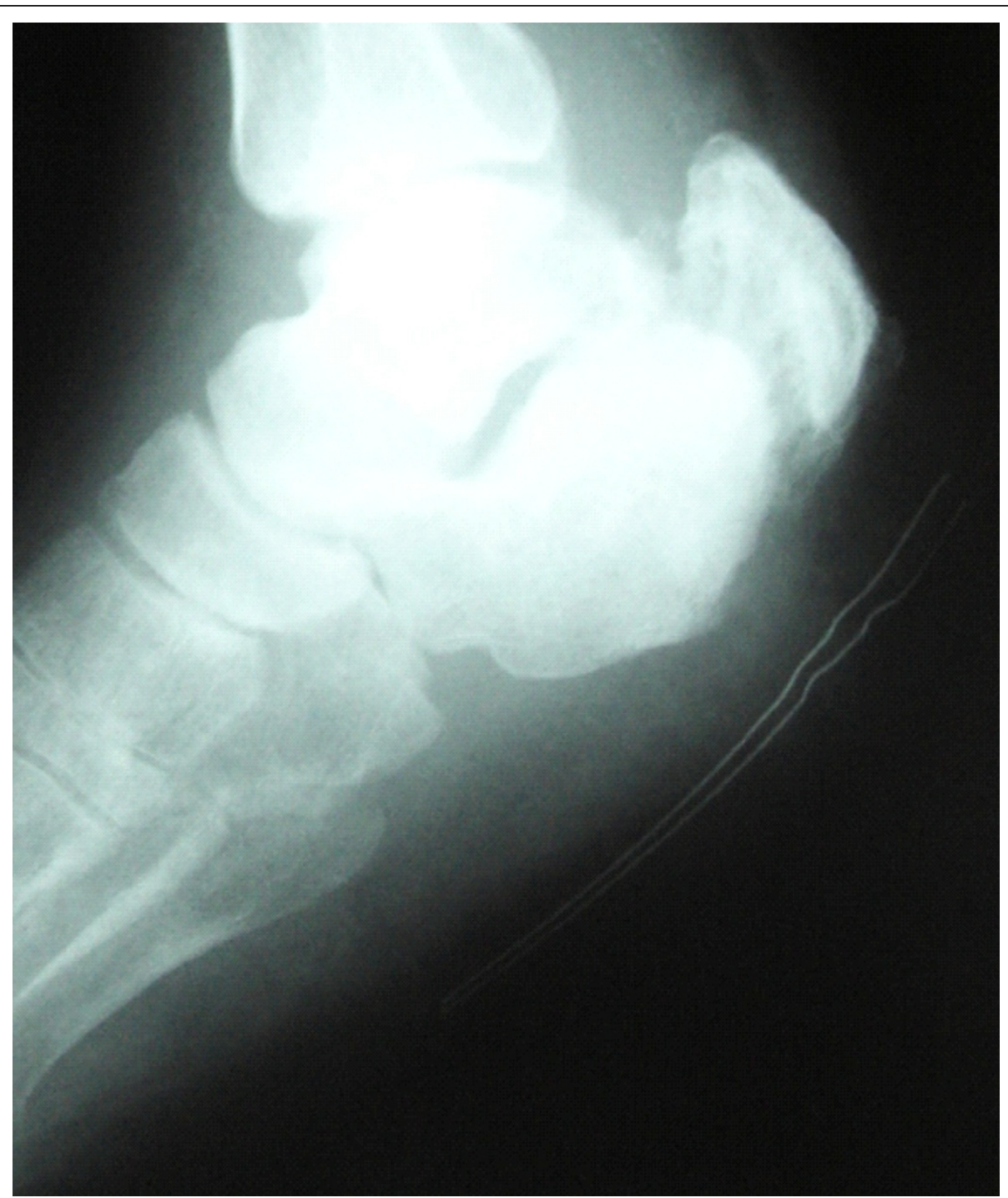

Figure 2 Standard Rx scan of the right heel at end of treatment.

osteomyelitis who were treated with Daptomycin at doses ranging between 3.2 and $7.5 \mathrm{mg} / \mathrm{Kg} /$ daily (median initial dose $5.6 \mathrm{mg} / \mathrm{kg}$ ) were retrospectively evaluated in the CORE 2004 database. Among clinically evaluable patients with osteomyelitis, $63 \%$ were cured. In $48 \%$ of them, Daptomycin was given concurrently with other antimicrobial agents; MRSA accounted for $45 \%$ of the identified pathogens. In another series, a $94 \%$ success rate was observed in patients treated with Daptomycin alone [26]. Although renal transplant recipients are most intensely immunosuppressed early in the posttransplantation period, immunosuppressive therapy could not be tapered in our patient over the past 3 years and during treatment. Therefore, the efficacy of Daptomycin, in conjunction with the repeated local debridement, may have played a major role.

\section{Conclusions}

Our experience adds to the large body of evidence that Daptomycin is effective and does not carry a relevant risk of renal damage in fragile patients as those with impaired renal function, renal transplant recipients or patients on nephrotoxic drugs. It could be effectively and safely used for MRSA osteomyelitis in a renal transplant recipient with recovered renal function. Higher dosing at 6 to $8 \mathrm{mg} / \mathrm{kg} /$ daily should be cautiously tested in the event of life-threatening infections, with tight monitoring of ensuing nephrotoxicity due to immunosuppressive agents $[27,28]$.

\section{Consent}

Written informed consent was obtained from the patient for publication of this Case report and any 
accompanying images. A copy of the written consent is available for review by the Editor-in-Chief of this journal.

\section{Abbreviations}

MRSA: Methicillin resistant Staphylococcus aureus; FAD: Fabry-Anderson Disease; DEXA: Dual-energy X-ray absorptiometry; VRE: Vancomycin-resistant Enterococcus.

\section{Acknowledgements}

We are sincerely indebted to all nurses of the Infectious Disease Unit, for their precious and constant assistance with the patient. Dr. E. Polilli was funded by an educational grant from the "Fondazione Camillo de Lellis per I'Innovazione e la Ricerca in Medicina", Pescara, Italy.

\section{Author details}

${ }^{1}$ Microbiology and Virology Unit, Pescara General Hospital, Pescara, Italy. ${ }^{2}$ Clinic of Infectious Diseases, G. D'Annunzio University, Chieti, Italy. ${ }^{3}$ Infectious Disease Unit, Pescara General Hospital, Pescara, Italy. ${ }^{4}$ Orthopedics Unit, Ortona Hospital, Ortona, Italy. ${ }^{5}$ Infectious Disease Unit, Pescara General Hospital, Via Fonte Romana, 8, Pescara 65124, Italy.

\section{Authors' contributions}

$E P, G P, A C$ and DD ideated this case-report and did most of the writing, supported by TU. VS, EM and MB have been involved in drafting the manuscript. FS has made substantial contributions to acquisition of data. GP has made substantial contributions to conception of the case redaction, have given final approval of the version to be published. All authors read and approved the final manuscript.

\section{Competing interests}

The authors declare that they have no competing interests.

Received: 5 January 2012 Accepted: 11 March 2012

Published: 11 March 2012

\section{References}

1. Deresinski S: Counterpoint: vancomycin and staphylococcus aureus-an antibiotic enters obsolescence. Clin Infect Dis 2007, 44:1543-1548.

2. Allison DC, Holtom PD, Patzakis MJ, Zalavras CG: Microbiology of bone and joint infections in injecting drug abusers. Clin Orthop Relat Res 2010, 468:2107-2112.

3. Brook I: Microbiology and management of joint and bone infections due to anaerobic bacteria. J Orthop Sci 2008, 13:160-169.

4. Lipsky BA, Weigelt JA, Gupta V, Killian A, Peng MM: Skin, soft tissue, bone, and joint infections in hospitalized patients: epidemiology and microbiological, clinical, and economic outcomes. Infect Control Hosp Epidemiol 2007, 28:1290-1298.

5. Mader JT, Mohan D, Calhoun J: A practical guide to the diagnosis and management of bone and joint infections. Drugs 1997, 54:253-264.

6. Sievert DM, Rudrik JT, Patel JB, McDonald LC, Wilkins MJ, Hageman JC: Vancomycin-resistant Staphylococcus aureus in the United States, 20022006. Clin Infect Dis 2008, 46:668-674.

7. Molin S, Tolker-Nielsen T: Gene transfer occurs with enhanced efficiency in biofilms and induces enhanced stabilisation of the biofilm structure. Curr Opin Biotechnol 2003, 14:255-261.

8. Nishimura S, Tsurumoto T, Yonekura A, Adachi K, Shindo H: Antimicrobial susceptibility of Staphylococcus aureus and Staphylococcus epidermidis biofilms isolated from infected total hip arthroplasty cases. J Orthop Sci 2006, 11:46-50.

9. Chamberlain RS, Culshaw DL, Donovan BJ, Lamp KC: Daptomycin for the treatment of surgical site infections. Surgery 2009, 146:316-324.

10. Rice DA, Mendez-Vigo L: Daptomycin in bone and joint infections: a review of the literature. Arch Orthop Trauma Surg 2009, 129:1495-1504.

11. Traunmuller F, Schintler MV, Metzler J, Spendel S, Mauric O, Popovic M, Konz KH, Scharnagl E, Joukhadar C: Soft tissue and bone penetration abilities of daptomycin in diabetic patients with bacterial foot infections. J Antimicrob Chemother 2010, 65:1252-1257.
12. Julian BA, Quarles LD, Niemann KM: Musculoskeletal complications after renal transplantation: pathogenesis and treatment. Am J Kidney Dis 1992, 19:99-120.

13. Sprague SM: Mechanism of transplantation-associated bone loss. Pediatr Nephrol 2000, 14:650-653.

14. Wang EH, Simpson S, Bennet GC: Osteomyelitis of the calcaneum. J Bone Joint Surg Br 1992, 74:906-909.

15. Mersebach H, Johansson JO, Rasmussen AK, Bengtsson BA, Rosenberg K, Hasholt L, Sorensen SA, Sorensen SS, Feldt-Rasmussen U: Osteopenia: a common aspect of Fabry disease. Predictors of bone mineral density. Genet Med 2007, 9:812-818.

16. Hiramatsu K: Vancomycin-resistant Staphylococcus aureus: a new model of antibiotic resistance. Lancet Infect Dis 2001, 1:147-155.

17. Garazzino S, Aprato A, Baietto L, D'Avolio A, Maiello A, De Rosa FG, Aloj D, Siccardi M, Biasibetti A, Masse A, Di Perri G: Glycopeptide bone penetration in patients with septic pseudoarthrosis of the tibia. Clin Pharmacokinet 2008, 47:793-805.

18. Norden CW, Shaffer M: Treatment of experimental chronic osteomyelitis due to staphylococcus aureus with vancomycin and rifampin. $J$ Infect Dis $1983,147: 352-357$

19. Lodise TP, Lomaestro B, Graves J, Drusano GL: Larger vancomycin doses (at least four grams per day) are associated with an increased incidence of nephrotoxicity. Antimicrob Agents Chemother 2008, 52:1330-1336.

20. Di Paolo A, Malacarne P, Guidotti E, Danesi R, Del Tacca M: Pharmacological issues of linezolid: an updated critical review. Clin Pharmacokinet 2010, 49:439-447.

21. Garcia-de-la-Maria C, Marco F, Armero $Y$, Soy D, Moreno A, del Rio A, Almela M, Cervera C, Ninot S, Falces C, Mestres CA, Gatell JM, Jiménez de Anta MT, Miró JM, Hospital Clínic Experimental Endocarditis Study Group: Daptomycin is effective for treatment of experimental endocarditis due to methicillinresistant and glycopeptide-intermediate Staphylococcus epidermidis. Antimicrob Agents Chemother 2010, 54:2781-2786.

22. Chaftari AM, Hachem R, Mulanovich V, Chemaly RF, Adachi J, Jacobson K, Jiang $Y$, Raad I: Efficacy and safety of daptomycin in the treatment of Gram-positive catheter-related bloodstream infections in cancer patients. Int J Antimicrob Agents 2010, 36:182-186.

23. Sader HS, Farrell DJ, Jones RN: Antimicrobial susceptibility of Grampositive cocci isolated from skin and skin-structure infections in European medical centres. Int J Antimicrob Agents 2010, 36:28-32.

24. Burkhardt $\mathrm{O}$, Kielstein JT: A simplified three-times weekly daptomycin dosing regimen for chronic hemodialysis patients. Expert Rev Anti Infect Ther 2010, 8:11-14

25. Kielstein JT, Eugbers C, Bode-Boeger SM, Martens-Lobenhoffer J, Haller H, Joukhadar C, Traunmüller F, Knitsch W, Hafer C, Burkhardt O: Dosing of daptomycin in intensive care unit patients with acute kidney injury undergoing extended dialysis-a pharmacokinetic study. Nephrol Dial Transplant 2010, 25:1537-1541.

26. Lamp KC, Friedrich LV, Mendez-Vigo L, Russo R: Clinical experience with daptomycin for the treatment of patients with osteomyelitis. Am J Med 2007, 120:S13-\$20.

27. Bassetti M, Nicco E, Ginocchio F, Ansaldi F, de Florentiis D, Viscoli C: Highdose daptomycin in documented Staphylococcus aureus infections. Int J Antimicrob Agents 2010, 36:459-461.

28. Seaton RA: Daptomycin: rationale and role in the management of skin and soft tissue infections. J Antimicrob Chemother 2008, 62(Suppl 3):15-23.

doi:10.1186/1476-0711-11-6

Cite this article as: Polilli et al:: Successful salvage therapy with Daptomycin for osteomyelitis caused by methicillin-resistant Staphylococcus aureus in a renal transplant recipient with FabryAnderson disease. Annals of Clinical Microbiology and Antimicrobials 2012 $11: 6$ 